\title{
Análisis Comparado de los Sistemas de Salud de la Región Andina y El Caribe
}

\author{
Diana Gómez-Camelo \\ Química Farmacéutica, M. Sc. Salud Pública, Investigadora Independiente. E-mail: \\ diana_gomez_camelo@yahoo.com.
}

Recibido 28 Marzo 2005/Enviado para Modificación 31 Mayo 2005/Aceptado 20 Septiembre 2005

\section{RESUMEN}

Objetivos Realizar un análisis comparado de los sistemas de salud de la región Andina y El Caribe que contribuya con la construcción de un panorama sobre las experiencias de los sistemas latinoamericanos.

Métodos Se utilizó la aplicación del análisis entre países (cross national), del método comparado. Se emprendió el análisis de los sistemas de salud de Bolivia, Colombia, Cuba, Ecuador, Perú, República Dominicana y Venezuela, entre el año 1990 y el año 2004. Se utilizó información documental de fuentes secundarias. Se realizó la comparación de las reformas y los cambios en el período mencionado así como de las configuraciones actuales de los sistemas. Se utilizaron tipologías descritas para el estudio de los sistemas de salud.

Resultados Se encontraron diferentes diseños organizacionales de los sistemas: un Sistema Nacional de Salud (SNS), sistemas segmentados y sistemas basados en el aseguramiento. En casi todos los sistemas la tendencia de las reformas de los años noventa y de las propuestas actuales se dirige hacia la adopción del aseguramiento de un paquete básico de servicios y el fortalecimiento de la competencia en la prestación con la participación de la mezcla pública y privada.

Conclusiones La organización y la estructura de la mayoría de los sistemas estudiados introdujeron y siguen introduciendo cambios de acuerdo con lineamientos internacionales. La generalidad de estas estructuras debe procurar aún por diseños que las fortalezcan como instrumentos que mejoren la calidad de vida de las poblaciones. El método comparado es una herramienta que permite acercarse al estudio de los sistemas de salud y genera información que puede alimentar el debate en los actuales procesos de reforma sectorial. Este trabajo se configura en la primera aproximación al estudio comparativo de los sistemas de salud de la Región Andina y El Caribe 
Palabras Clave: Estudio comparativo, reforma del sector salud, sistema de salud, América Latina, América del Sur, región del Caribe (fuente: DeCS, BIREME).

ABSTRACT

Comparative analysis of Andean and Caribbean region health systems

Objective Carrying out a comparative analysis of Andean and Caribbean health systems contributing towards the general panorama of Andean and Caribbean region health care system experience.

Methods This study was aimed at carrying out a comparative analysis of health systems in Bolivia, Colombia, Ecuador, Peru, Venezuela, the Dominican Republic and Cuba between 1990 and 2004. Documentary information from secondary sources was used. Reform and changes during the aforementioned period were compared, as well as the systems' current configurations. Described typologies were used for studying the health systems.

Results Different organisational designs were found for the systems: a national health system (NHS), segmented systems and systems based on mandatory insurance. The trend of reforms introduced in the 1990s and current proposals in almost all systems are directed towards adopting mandatory insurance via a basic packet of services and strengthening competition in providing services through a public and private mix.

Conclusions The organisation and structure of most systems studied have introduced and continue to introduce changes in line with international guidelines. The generality of these structures means that efforts must still be made to adopt designs strengthening them as instruments improving populations' quality of life. Comparative analysis is a tool leading to studying health systems and producing information which can nourish debate regarding current sector reform. This work took shape during the first approach to a comparative study of Andean region and Caribbean health systems.

Key Words: Comparative study, health care reform, health care system, Latin-America, South America, Caribbean region (source: MeSH, NLM).

L

as reformas y los cambios realizados durante los últimos quince años a los sistemas de salud de América Latina, no han logrado los objetivos con los cuales fueron implantados en términos de disminución de inequidades, uso eficiente de recursos, y aumento de la calidad de los servicios. Algunos de estos cambios y reformas siguieron los lineamientos internacionales propuestos por el Banco Mundial, a través de los documentos "Financing Health Services in Developing Countries: An Agenda for Reform" de 1987 y el "Informe Sobre el Desarrollo Mundial 1993: Invertir en Salud”. La Organización Panamericana de la Salud (OPS) (1), ha reconocido que estas transformaciones no han producido un efecto global positivo sobre el sector salud. Bajo este panorama, actualmente los países latinoamericanos 
reconocen la necesidad de diseñar y realizar nuevas reformas en sus sistemas. Es aquí, donde la generación de información comparada de las diferentes experiencias nacionales en la región, puede generar importantes elementos de análisis. El hecho de realizar comparaciones implica reconocer similitudes y diferencias, que es la esencia misma del método, con otros sistemas diferentes, lo que facilita, desde un panorama más amplio, un mejor entendimiento de la experiencia propia.

\section{MÉTODO}

Para Pliscoff (2), el hecho de involucrase en un estudio comparativo sobre cualquier ámbito en que se mueve la sociedad, necesita tener claro la sistematización del método puesto que no se conocen en profundidad realidades distintas y complejas a priori. El enfoque analítico del presente estudio se basó en una aplicación del método comparado denominado: análisis entre países (cross-national), que incluye características de las estrategias comparativas del análisis de casos y de variables (3); Realizar una comparación utilizando el enfoque del análisis entre países implica: 1) utilizar un marco conceptual y un sistema de análisis que permita la comparación, 2) definir los conceptos operativos y 3) seleccionar las unidades de análisis (3).

Marco conceptual y sistema de análisis: La Organización Mundial de la Salud (OMS) ha definido un sistema de salud como el conjunto de las organizaciones, instituciones y recursos dedicados a la actividad sanitaria, lo que involucra a los servicios de salud personales, los servicios de salud pública o el desempeño de una actividad intersectorial (4). En el desarrollo de este trabajo, los sistemas de salud se entenderán en un sentido más estrecho, que involucra sólo a los servicios de salud.

De otro lado y en medio de un importante debate, se han propuesto diversas tipologías para acercarse al estudio de los sistemas de salud. Las tipologías presentadas en la literatura se basan en enfoques conceptuales y metodologías distintas, que seleccionan diferentes características para la clasificación y estudio de los sistemas. Aquí se utilizan cuatro grandes tipologías de los sistemas: a) Oferta y Demanda, b) Naturaleza de los bienes (público, privado o la mezcla de ambos), c) Financiamiento (recaudo fiscal; cotizaciones; y pagos privados a seguros y gastos de bolsillo) y d) Organización y Estructura. Con respecto a este último, la organización y estructura se refiere a un conjunto de características de los sistemas como la forma de recaudo de los recursos económicos, la manera de asegurar la provisión de los servicios y los arreglos institucionales para el desempeño de las funciones, como la integración o desintegración institucional. De esta manera se ha propuesto por 
ejemplo, la caracterización de un Sistema Nacional de Salud o de un Seguro Nacional de Salud.

Conceptos operativos: Estos se utilizaron tanto para la descripción como para la comparación de unidades. Para la descripción se usaron las siguientes categorías con sus correspondientes conceptos: a) El contexto (político, económico, epidemiológico, demográfico y social) y b) La organización de los sistemas, que incluyó: las reformas y los cambios realizados durante la década de los años noventa y la organización actual (el financiamiento, la prestación, el gasto en salud, el aseguramiento y la cobertura). Para la comparación entre unidades se utilizó únicamente la organización de los sistemas, lo que abarca los conceptos anteriormente mencionados.

Selección de países: Una de las herramientas del método comparado para la selección es la definición de unidades similares y diferentes. Con la clasificación de entidades similares se pretende mantener constantes el mayor número de variables como sea posible (3), como el nivel de desarrollo, la cultura y el modelo político. Se consideraron entidades similares los países Andinos (Bolivia, Colombia, Ecuador, Perú y Venezuela) y República Dominicana. Como entidad diferente se consideró a Cuba. Esta caracterización se basa en la naturaleza de su sistema político y por ende económico.

Se utilizó información de fuentes secundarias como la generada por los organismos de cooperación técnica como la OPS, OMS, Fondo de las Naciones Unidas para la Infancia (UNICEF), Programa de las Naciones Unidas para el Desarrollo (PNUD), Population Reference Bureau (PRB), información institucional de fuentes nacionales, artículos académicos, leyes y decretos nacionales.

\section{RESULTADOS}

A pesar de las similitudes entre los países, con excepción de Cuba, se presentan diferencias nacionales que impactan en su situación de salud, como son: los contextos demográficos, socioeconómicos y epidemiológicos (Tabla 1), así como la organización de los sistemas de salud particulares de cada país.

Con respecto a la comparación de los sistemas se tocarán puntos relevantes, como son: las reformas de la última década, la organización actual, la relación del gasto de salud con ciertos indicadores de salud y las convergencias y divergencias en cuanto a las tendencias de los diseños organizacionales de los sistemas. 
Tabla 1. Algunos indicadores demográficos, socioeconómicos y epidemiológicos

\begin{tabular}{|c|c|c|c|c|c|c|c|}
\hline Indicadores & Bolivia & Colombia & Cuba & Ecuador & Perú & $\begin{array}{c}\text { República } \\
\text { Dominicana }\end{array}$ & Venezuela \\
\hline $\begin{array}{l}\text { Población Total, } \\
\text { en millones (13) }\end{array}$ & 8,6 & 44,0 & 11,2 & 13,0 & 27,0 & 9,0 & 25,0 \\
\hline $\begin{array}{l}\text { Crecimiento anual } \\
\text { de la población, } \\
\text { en } \%(14)\end{array}$ & 2,3 & 1,8 & 0,4 & 2,0 & 1,7 & 1,7 & 2,0 \\
\hline $\begin{array}{l}\text { Proporción de } \\
\text { Población Ur- } \\
\text { bana, en \% (14) }\end{array}$ & 63,5 & 76,0 & 75,7 & 64,0 & 73,5 & 66,6 & 87,4 \\
\hline $\begin{array}{l}\text { Pobreza, \% vive } \\
\text { con menos de } 1 \\
\text { USD diario } \\
\text { (14) }\end{array}$ & 14,4 & 19,7 & - & 20,2 & 15,5 & 3,2 & 23,0 \\
\hline $\begin{array}{l}\text { Coeficiente de } \\
\text { Gini (16) }\end{array}$ & 44,7 & 57,1 & - & 43,7 & 46,2 & 47,4 & 49,5 \\
\hline $\begin{array}{l}\text { PIB per cápita en } \\
\text { dólares } \\
\text { internacionales } \\
\text { (18) }\end{array}$ & 2,370 & 6,519 & 3,168 & 3,905 & 4,888 & 5,792 & 6,402 \\
\hline $\begin{array}{c}\text { Esperanza de } \\
\text { Vida al nacer (15) }\end{array}$ & 64 & 72 & 77 & 71 & 70 & 67 & 74 \\
\hline $\begin{array}{l}\text { Mortalidad } \\
\text { materna, } \\
\text { por } 100.000 \\
\text { nacidos vivos (14) }\end{array}$ & 550 & 120 & 24 & 210 & 240 & 110 & 65 \\
\hline $\begin{array}{l}\text { Tasa mortalidad } \\
\text { menores de } 1 \\
\text { año, por } 1000 \\
\text { nacidos vivos (15) }\end{array}$ & 53 & 18 & 6 & 24 & 26 & 29 & 18 \\
\hline $\begin{array}{l}\text { Gasto en salud } \\
\text { como \% del PIB } \\
\text { (19) }\end{array}$ & 5,47 & 9,30 & 6,68 & 3,99 & 4,35 & 6,51 & 8,77 \\
\hline $\begin{array}{l}\text { Gasto total per } \\
\text { cápita en salud } \\
\text { en dólares } \\
\text { internacionales } \\
(18)\end{array}$ & 125 & 356 & 229 & 177 & 231 & 353 & 386 \\
\hline
\end{tabular}

\section{Reformas}

Con excepción de Cuba, el período de reformas sanitarias y cambios en estos países durante la década de los años noventa, estuvo inmerso dentro de un contexto marcado por una crisis económica, seguida por políticas de ajuste estructural y transformación del papel del Estado, donde la banca multilateral tuvo grandes influencias, así como los intereses específicos de los grupos dominantes en cada país. 
Los cambios en Bolivia, Ecuador, Perú y Venezuela no han sido cortos en el tiempo a la vez que no se ha dado una transformación verdaderamente estructural de los sistemas de salud, sino que se han hecho arreglos institucionales con la instauración de nuevos elementos. Es así, como se han introducido seguros públicos, dirigidos a poblaciones vulnerables y de alto riesgo epidemiológico como en Bolivia, Ecuador y Perú.

En Perú se permitió y promovió la participación del sector privado en la afiliación y prestación de servicios de salud dirigidos a los usuarios del seguro social. Comparativamente, de los países mencionados, es Ecuador la nación que normativamente ha introducido mayores cambios que obedecen a las tendencias internacionales. Este sistema se orienta hacia el aseguramiento universal de la población mediante la cobertura de un plan de salud que contiene un paquete básico de prestaciones individuales. Se estipula la rectoría pública y la participación en la prestación de servicios del sector público y del privado. Aún así, en este país no se tiene reglamentada la operación de aseguradores, ni la clara separación de funciones, como tampoco un fondo de financiamiento único.

En Venezuela, en 1998, se aprobaron las leyes de la Seguridad Social donde se introducía la competencia en la administración de los servicios sanitarios bajo una concepción de mercado de estos servicios, sin embargo estas leyes fueron derogadas en el marco de los cambios políticos ocurridos en este mismo año y en 1999 con la aprobación de la nueva constitución Bolivariana. De esta manera se retomó la prioridad de la propiedad pública, el subsidio a la oferta, el financiamiento y la prestación de servicios a cargo de una misma institución; lo que deja al sistema de salud bajo el mismo diseño organizacional que antes de la década de los años noventa.

En República Dominicana y Colombia, la reforma en el sistema de salud, fue rápida en el tiempo a la vez que involucró serias modificaciones en la estructura, su organización y por tanto en el financiamiento y la prestación de los servicios.

Los procesos de cambio en Cuba estuvieron dirigidos a mejorar la eficiencia, la calidad y a garantizar la sostenibilidad financiera del sistema. Estos cambios no se presentaron como una oleada influida por la banca multilateral, ni por la forma en que se inserta la economía de este país, en la economía del mundo; sino como un proceso continuo en el tiempo, bajo la misma lógica con la que fue creado el Sistema Nacional de Salud cubano. 
Organización Actual

A continuación se presenta en forma esquemática el uso de las tipologías propuestas para la caracterización de los sistemas:

Tabla 2. Organización y resultados de los sistemas

\begin{tabular}{|c|c|c|c|}
\hline Componentes & $\begin{array}{c}\text { Bolivia } \\
\text { Ecuador } \\
\text { Perú } \\
\text { Venezuela }\end{array}$ & $\begin{array}{l}\text { Colombia } \\
\text { Republica } \\
\text { Dominicana }\end{array}$ & Cuba \\
\hline $\begin{array}{l}\text { - Integración } \\
\text { Institucional: }\end{array}$ & No & No & $\mathrm{Si}, \mathrm{SNS}$ \\
\hline - Articulación & No & $\mathrm{Si}$ & $\mathrm{Si}$ \\
\hline $\begin{array}{l}\text { - Segmentación } \\
\text { Poblacional }\end{array}$ & Si & $\mathrm{Si}$ & No \\
\hline Oferta / Demanda & Oferta y demanda & $\begin{array}{c}\text { Demanda, coexiste } \\
\text { financiamiento a la } \\
\text { oferta. }\end{array}$ & Oferta \\
\hline Bienes y Servicios & Público y privado & $\begin{array}{l}\text { Mezcla pública - } \\
\text { privada }\end{array}$ & Público \\
\hline $\begin{array}{l}\text { Fuentes de } \\
\text { Financiamiento }\end{array}$ & $\begin{array}{l}\text { 1) Recaudo fiscal } \\
\text { (Subsector público) } \\
\text { 2) Cotizaciones } \\
\text { (Seguridad Social) } \\
\text { 3) Seguros } \\
\text { Privados / gastos } \\
\text { Bolsillo (Subsector } \\
\text { privado) }\end{array}$ & $\begin{array}{l}\text { Cotizaciones y } \\
\text { recaudo fiscal }\end{array}$ & Presupuesto estatal \\
\hline $\begin{array}{l}\text { Financiamiento y } \\
\text { Prestación }\end{array}$ & Público y Privado & $\begin{array}{l}\text { Financiamiento } \\
\text { público. Mezcla } \\
\text { pública privada en } \\
\text { la prestación. }\end{array}$ & Público \\
\hline
\end{tabular}

Se identificaron y compararon otras características de los sistemas, las cuales se describen en la Tabla 3.

Relación entre el gasto e indicadores de salud

Si bien es cierto, los resultados de indicadores como la expectativa de vida, la mortalidad materna e infantil y la situación epidemiológica de un país no son resultado directo de los servicios de salud, observar la relación de éstos con el gasto en salud que hace un país, puede señalar aspectos generales de cómo se usan los magros recursos de países con economías medias como los de este estudio. 
Tabla 3. Otras Características de los Sistemas

\begin{tabular}{|c|c|c|c|}
\hline Características & $\begin{array}{c}\text { Bolivia } \\
\text { Ecuador } \\
\text { Perú } \\
\text { Venezuela }\end{array}$ & $\begin{array}{c}\text { Colombia } \\
\text { Republica Dominicana }\end{array}$ & Cuba \\
\hline $\begin{array}{c}\text { Aseguramiento } \\
\text { (Seguros Públicos) }\end{array}$ & $\begin{array}{l}\text { Seguros focalizados } \\
\text { en población } \\
\text { vulnerable. (Excepto } \\
\text { Venezuela) }\end{array}$ & $\begin{array}{l}\text { Seguro Obligatorio } \\
\text { (paquete de } \\
\text { beneficios) }\end{array}$ & $\begin{array}{c}\text { Todas las } \\
\text { prestaciones }\end{array}$ \\
\hline Principios de Ingreso & $\begin{array}{c}\text {-Pobreza (subsector } \\
\text { público) } \\
\text {-Mérito } \\
\text { Reconocido } \\
\text { (Seguridad Social) } \\
\text { - Poder de } \\
\text { Compra (Subsector } \\
\text { privado) }\end{array}$ & $\begin{array}{c}\text { - Pobreza } \\
\text { - Mérito reconocido. } \\
\text { - Poder de compra } \\
\text { (para planes } \\
\text { complementarios) }\end{array}$ & Ciudadanía \\
\hline Modelo de Atención & $\begin{array}{c}\text { Curativo } \\
\text { Reparativo }\end{array}$ & $\begin{array}{c}\text { Curativo } \\
\text { Reparativo }\end{array}$ & $\begin{array}{c}\text { Basado en APS. } \\
\text { Médico y } \\
\text { enfermera de } \\
\text { familia }\end{array}$ \\
\hline Cobertura & No universal & No universal & Universal \\
\hline
\end{tabular}

Los países muestran un comportamiento heterogéneo entre sí (Tabla 1). Existe una brecha importante para la expectativa de vida, la mortalidad infantil y materna entre Cuba y Bolivia, mientras que entre estos dos extremos de la comparación, se ubican los resultados de los restantes países. Con respecto al gasto en salud como porcentaje del PIB, se encontraron cifras cercanas al de países desarrollados, $10 \%$, como en el caso colombiano; mientras que países como Ecuador y Perú presentan datos que indican al menos la mitad de este valor. Para el gasto total per cápita en salud, se presentan diferencias que van desde 125 dólares internacionales en Bolivia, hasta gastos tres veces mayores que éste como en Colombia y Venezuela.

Para el caso particular, los datos utilizados tanto para los indicadores de salud como de gasto, resumen la tendencia temporal de al menos un lustro para cada uno de los países.

Al analizar simultáneamente varios indicadores, la evidencia muestra que los países con los mayores PIB per cápita como Colombia y Venezuela (Tabla 1), presentan mayor porcentaje de éste como gasto en salud y con respecto a los gastos totales per cápita. La tendencia es similar para Ecuador y Bolivia, es decir, siendo países con bajo PIB per cápita comparado con los restantes países, sus gastos como porcentaje del PIB y los gastos totales per cápita también son los menores. Sin embargo, observando los datos para Cuba, se puede percibir que si bien la cantidad de recursos invertida en el 
sector salud puede depender de cierta medida de la cantidad de recursos totales de una nación, esta relación no es directa, puesto que también depende de la prioridad que tengan las políticas sectoriales en salud en un país.

También se encontró evidencia sobre que el monto total invertido en salud no es directamente proporcional a los resultados de los indicadores de salud; es decir, los resultados en salud no son mejores en la medida del aumento del gasto. Es así como algunos países, Colombia, con un alto gasto en salud, muestra resultados en sus indicadores de salud que tienen mucho por mejorar, sumado con el hecho de que este país ha reportado serios problemas en la última década en relación al deterioro de los programas de salud pública (vigilancia epidemiológica, disminución de las tasas de vacunación, entre otros). De otro lado, un país como Cuba, con una tendencia de recursos nacionales menores y un relativo bajo gasto sanitario como porcentaje del PIB comparativamente hablando, tiene unos muy buenos resultados de los indicadores salud, así como resultados más directos del sistema sanitario, como altas tasas de vacunación, se erradicó la malaria desde la década de los años setenta, el sarampión en los noventa y no se ha registrado incidencia de casos de Cólera desde hace varias décadas.

\section{Convergencias y divergencias}

Con base en las actuales propuestas de reforma en los países estudiados, la organización de estos sistemas se dirige hacia el aseguramiento universal de un conjunto de prestaciones básicas, una provisión mixta (público - privado), administración pública del financiamiento, regulación y rectoría estatal. Sin embargo en Venezuela, la oposición frente a los lineamientos internacionales de reforma, ha servido como bandera de resistencia política, lo que ha dirigido al sistema de este país hacia una tendencia organizacional distinta que en los restante países andinos, pero no así en la situación real. De esta manera, en Ecuador, Perú, Bolivia, Colombia y República Dominicana, se tiende a configurar el mercado de los servicios médicos regido por las leyes de la oferta y la demanda.

Como un sistema divergente de estas directrices se encuentra Cuba, donde el Sistema Nacional de Salud es público y el Estado regula, financia y presta servicios de salud, sumado al hecho de que la salud se configura como un derecho social. Es importante anotar que además de las diferencias de su sistema político, este país no comparte con los anteriores, presiones tan directas de la banca multilateral por el cumplimiento de pagos de la deuda externa, aunque presenta otras profundas presiones políticas y económicas. 


\section{DISCUSIÓN}

A través del presente ejercicio de comparación se obtuvo un mayor conocimiento sobre las directrices de los sistemas seleccionados y las relaciones con los contextos extrasectoriales. Dentro de las limitaciones más importantes en el desarrollo de este estudio se encuentran: el uso de datos estadísticos secundarios reproduce las inconsistencias derivadas de las fuentes primarias; las medidas promedio que se seleccionaron no expresan el comportamiento de las diferencias y desigualdades existentes al interior de los países; escasa disponibilidad de información actualizada; existen grandes diferencias entre los datos reportados por las agencias de cooperación técnica como la OPS o la OMS y los reportados por fuentes nacionales de tipo académico.

La aplicación del método comparado y su estrategia de análisis entre países, sumado con el uso de tipologías y otras características, más que generalizar o encontrar causalidades de los fenómenos encontrados, permitió describir las configuraciones de los sistemas, y proporcionó herramientas para comprender esas configuraciones dentro de unos contextos nacionales propios, y dentro de tendencias extrasectoriales internacionales, asimismo permitió identificar similitudes, diferencias, convergencias y divergencias.

De esta manera, el uso de la aplicación del análisis entre países, es una ruta que permite mostrar evidencias e interpretaciones que no pueden ser percibidas en el estudio de un solo sistema. Es así como se encontraron varias configuraciones de los servicios de salud en los países estudiados, que van desde un Sistema Nacional de Salud como el cubano, pasando por sistemas basados en el aseguramiento individual de un paquete de servicios como el colombiano y dominicano, y sistemas segmentados como el boliviano, ecuatoriano, peruano y venezolano. Estas configuraciones permiten evidenciar los diferentes grados de participación y control del Estado en las funciones de los sistemas.

Con respecto al tipo de seguros ofrecidos por el Estado, en el sistema de salud cubano se aseguran todas las prestaciones; en los sistemas de salud de Colombia y República Dominicana, el Estado ofrece un seguro nacional de salud diseñado al priorizar las necesidades de la población y además se presenta la coexistencia de planes complementarios de salud y medicina prepagada que funcionan bajo lógica de los seguros privados. En Bolivia, Perú y Ecuador la figura del seguro público cobija a las poblaciones focalizadas por riesgo. 
El único sistema que cumple con la cobertura legal es Cuba, el cual presenta una cobertura real de $100 \%$, lo que lo caracteriza como de cobertura universal. En el resto de países, la población sin acceso está entre un 20-30 \%. Con respecto al aseguramiento, en Bolivia y Ecuador la población sin asegurar es cercana al 85\%, mientras que tanto en Venezuela como en Colombia es del 35- $40 \%$, a pesar de las diferencias en las lógicas de organización de los servicios sanitarios en estos dos últimos países.

Basados en las condiciones actuales donde entre otros problemas no se ha logrado un sistema de cobertura universal, equitativo y de calidad (excepto en Cuba), todos los países reconocen la necesidad de introducir nuevas reformas en sus sistemas. Estas abarcan desde los proyectos actuales para superar los problemas del sistema, como en Colombia, hasta la introducción de la figura del aseguramiento universal de un paquete de servicios, como en Ecuador; además se incentiva la competencia entre el sector público y privado por la prestación de servicios, como en Perú y Ecuador, y la focalización de población vulnerable, como en Bolivia y Perú.

Utilizar el método comparado, en el estudio de los sistemas de salud, proporciona perspectivas amplias que llevan finalmente a conocer y examinar el propio sistema de salud desde la vista de otros y da argumentos para analizar las posibilidades y potencialidades que tienen los sistemas de salud para funcionar como una verdadera herramienta que permita mejorar la salud de la población y su calidad de vida

\section{REFERENCIAS}

1. Organización Panamericana de la Salud. Análisis de las reformas del sector salud en los países de la región Andina. Iniciativa Regional de Reforma del Sector de la Salud en América Latina y El Caribe; Washington DC: OPS; 2003.

2. Pliscoff C, Monje P. Método comparado: un aporte a la investigación en gestión pública. VIII Congreso Internacional del CLAD sobre la Reforma del Estado y de la Administración Pública. Panamá: Octubre, 2003.

3. Cais J. Metodología del análisis comparativo. Cuadernos Metodológicos No 21. Madrid: CIS; 1997.

4. Granados R, Gómez M. La reforma de los sistemas de salud en Chile y Colombia: resultados y balance. Revista de Salud Pública 2002; 2:97-120.

5. Le Bonniec Y. Sistemas de protección social comparados: Colombia, Brasil y México. Revista de Salud Pública 2002; 4(3):203-239.

6. Restrepo M. La reforma a la seguridad social en salud de Colombia y la teoría de la competencia regulada. En: Titelman D, Uthoff A (comp). Ensayos sobre el financiamiento de la seguridad social en salud. Los casos de: Estados 
Unidos, Canadá, Argentina, Chile, Colombia. Volumen II. Santiago de Chile: CEPAL; 2000.

7. Frenk J, Donabedian A. Intervención del Estado en la atención médica: Tipos, tendencias y variables. En: White K (ed). Investigaciones sobre servicios de salud: Una antología. Washington, DC.: OPS; 1992. p 1082 - 1097.

8. Soberón GA, Narro J. Equidad y atención de salud en América Latina. Principios y Dilemas. Boletín de la Oficina Sanitaria Panamericana 1985; 99(1):1-9.

9. Vargas I, Vásquez M, Jane E. Equidad y reformas de los sistemas de salud en Latinoamérica. Cadernos de Saúde Pública 2002; 18(4):927-937.

10. Martínez P, Rodríguez L, Agudelo C. Equidad en la política de reforma del sistema de salud. Revista de Salud Pública 2001; 3(1):13-39.

11. De los Santos-Briones S, Garrido-Solano C, Chávez-Chan M. Análisis comparativo de los sistemas de salud de Cuba y Canadá. Revista Biomedica 2004; 15(2):81-91.

12. González-Rossetti A, Bossert T. Mejorando la factibilidad política de la reforma en salud: un análisis comparativo de Chile, Colombia, y México. Washington: Iniciativa regional de reforma del sector salud en América Latina y el Caribe; 2000.

13. Population Reference Bureau. Cuadro de la población mundial 2003. Washington DC: PRB 2003.

14. Population Reference Bureau. Country Profiles for Population and Reproductive Health: Policy Developments and Indicators 2003. Washington DC: PRB; 2003.

15. Fondo de las Naciones Unidas para la Infancia. Estado Mundial de la Infancia 2005. Nueva York: UNICEF; 2004.

16. Programa de las Naciones Unidas para el Desarrollo. Informe Sobre Desarrollo Humano 2003. Indicadores de desarrollo humano. Nueva York: PNUD; 2003.

17. World Bank [Internet]. World Development Indicators database 2004. Disponible en: http://www.worldbank.org. Consultado Mayo de 2004.

18. Organización Mundial de la Salud [Internet]. Core Health Indicators. Disponible en: http://www.who.int/countries/es/. Consultado Mayo de 2002.

19. Pan American Health Organization. Health Expenditures for Latin America and the Caribbean. Division of health and human development public policy and health program. Washington DC: PAHO; 2002. 